\title{
OPINION
}

\section{The 2015 Nepal Earthquake Disaster: Is the Threat of Occurrence of Communicable Disease Epidemic Over?}

\author{
Sakiko Kanbara ${ }^{1,2}$, Nlandu Roger Ngatu ${ }^{1,2^{*}}$, Tara Pokhrel T ${ }^{3}$, Apsara Pandey ${ }^{3}$, \\ Chandrakara Sharma ${ }^{2}$, Hyeon J. Lee ${ }^{1,2}$, Shoko Miyagawa ${ }^{4}$, Hiroyuki Miyazaki ${ }^{5}$, Sayumi \\ Nojima $^{1,2}$, Nursing Association of Nepal (NAN) ${ }^{2}$ \\ ${ }^{1}$ Graduate School of Health Sciences \& Nursing, University of Kochi, Kochi, Japan; ${ }^{2}$ Disaster \\ Nursing Global Leading doctoral program (DNGL), University of Kochi, Kochi, Japan; ${ }^{3}$ Tribhuvan \\ University, Kathmandu, Nepal; ${ }^{4}$ Keio University, Tokyo, Japan; ${ }^{5}$ Asian Institute of Technology, \\ Bangkok, Thailand.
}

(Received: January 2018/ Revised: January 2018/ Accepted: February 2018)

\begin{abstract}
This opinion paper highlights the state of public health assessment in evacuation centers following the 2015 Nepal earthquake. It also suggests an approach to reinforce risk assessment and surveillance of communicable diseases (CD) in remote Nepalese districts. A short surveillance research was conducted on outbreaks of infectious diseases in Nepal in the post-2015 earthquake in evacuation centers in Kathmandu and Dhading districts. In collaboration with the Nursing Association of Nepal (NAN), the researchers have established a monitoring and surveillance system, named 'EpiNurse' program, in remote Nepalese districts. Periodic shelter to shelter visits, CD risk assessment and relief needs inventory in local communities are implemented, whereas health events with a potential to cause a CD outbreak are being reported to governmental agencies and health clusters involved in post-disaster relief in Nepal. Several cases of diarrheal diseases were identified in Nepalese districts after the 2015 earthquake, suggesting the existence of potential risk for the occurrence of new CD epidemics. Onsite risk assessment and monitoring of the effectiveness of actions and interventions implemented, as well as improvement of risk communication between relief agencies should be expanded to less resourced districts to reduce the risk of CD outbreak occurrence.
\end{abstract}

Keywords: Communicable disease; disaster risk reduction; earthquake; epidemics; Nepal

\section{Introduction}

An occurrence of a primary disaster of great magnitude often results in subsequent emergence of various health problems due to changes in the living environment. Communicable diseases (CD), for example, are common in displaced populations in the aftermath of a large scale disaster, especially due to poor access to basic needs, such as adequate shelter, safe water, and sanitation; sufficient nutrition; and primary healthcare services (Uprety et al., 2017). The relationship between the occurrence of a disaster and potential outbreak of infectious diseases is reported to be associated with the size and the characteristics of the displaced populations. In developing countries, the lack of adequate infrastructures and resources, the inexistence of disaster preparedness programs are among factors that contribute to increased risk of epidemics following a humanitarian crisis (Watson, Gayer, \& Connolly, 2007; Noji, 1997; Michel et al., 2007). Additionally, malnutrition represents an important public health issue (Spiegel, 2005), as it weakens immunity and increases vulnerability to infectious agents. This paper highlights the current state of public health assessment in temporary shelters 
and evacuations centers in Nepal, following the 2015 earthquake, enriched with field experience of the authors, and suggests ways to reinforce CD risk assessment and surveillance particularly in remote Nepalese districts.

\section{Risk and post-disaster epidemiologic profile of infectious diseases in Nepal}

Pre-existing cases of diarrheal diseases. When a pathogen responsible of a CD is present in disaster-affected area, there is a risk of large scale outbreak, given the deterioration of hygiene and sanitation as a result of the catastrophic event. In Nepal, for example, cholera has been reported since the 1960's, and even prior to the recent disaster (Gautam et al., 2012; Abou-Gareeb, 1961). Thus Vibrio cholerae, the pathogen that causes Cholera, is present in most Nepalese districts, including the capital Kathmandu. In 1961, a first report from Abou-Gareeb on a Cholera epidemic in Nepal was published by the Bulletin of the World Health Organization, which affected more than 3,000 people, killing about 405 of them in Kathmandu. This epidemic has reached other districts such as Bathkapore, Patan, Morang, Dhankuta districts etc. (Abou-Gareeb, 1961). On the other hand, the Haitian 2010 post-earthquake cholera epidemic, the worst outbreak in the history of this disease, has been reported to be imported from Nepal through Nepalese UN Peacekeepers (Katz, 2010). In addition, human rotaviruses and other pathogens linked to cases and episodes of diarrheal disease epidemics in Nepal have been reported (Uchida et al., 2006; Bhandari et al., 2015). The World Health Organization (WHO) estimates that diarrheal diseases account for 3.3\% of annual mortality rate in Nepal (WHO, 2015). Moreover, a report released by the Health Cluster Surveillance Team in Nepal (Health cluster bulletin, May 2015) mentioned 35 cases of diarrheal diseases in Sindhuli district.

Vector-borne diseases. Parasitic and viral infections are common in most developing countries, particularly in tropical regions. Of the diseases transmitted by mosquitoes, malaria and dengue fever are among the most prevalent illnesses in those countries. A change in the ecology due to a large scale disaster or adverse meteorological conditions (rainfall, humidity) may increase disease vectors' population and the risk of disease transmission among disaster-affected populations. Given that insects that transmit some viruses to human are present in almost all Nepalese districts, increased vector breeding sites might influence the prevalence of those illnesses. Moreover, the presence of rodents in shelters or evacuees' dwelling places may also be a source of contamination by transmissible pathogens.

\section{Trend of waterborne infectious disease in the post 2015 Nepalese earthquake}

Recent reports have shown limited water supply, sanitation and hygiene resources and a marked increase the incidence of waterborne infections in the post 2015 Nepal earthquakes (Simkhada et al., 2015; Uprety et al., 2017). According to the Nepalese Department of Health Services (DOHS, 2016), compared to the years 2013-2014, there was a $80 \%$ increase in communicable waterborne infection cases in 2015, particularly two months after the April earthquake disaster.

\section{Communicable diseases and human behavior on disaster}

The Division of Health Services of the Nepalese government published in their Weekly Bulletin called 'Early Warning and Reporting System (EWARS)' that there was no death by cholera or any other communicable diseases in the disaster affected areas in Nepal (Government of Nepal, 2015). However, all the data obtained were from district hospitals, not medical staff working in shelters. 
In contrast to this report from the government, researchers found various cases of syndrome and death related to com municable diseases in shelters. During the implementation of EpiNurse program, shelter situations were chaotic. Rapid health monitoring for communicable disease was conducted during mobil clinic visits by EpiNurse team members. Following the 2015 earthquake in Nepal, relief teams offered consultations and assistance to communities concerning syndromes in disasteraffected districts mostly within a 3-month period, during the acute phase of the disaster. However, it is known that various post-disaster problems and health issues often occur, which may overlap and fluctuate; such problems should neither be considered individually nor in isolation, particularly when unexpected health risks arise.

To reduce the risk of communicable disease epidemic, a greater attention should be paid in regard to hygiene control in ord e r to maintain acceptable health safety level in shelters. Reducing risks implies behavioral and lifestyle changes during a temporary settlement period after disaster. Additionally, a "people-centered" approach to disaster risk requires human behavioral consideration on how people interpret and live with the risks, and how their individual perception, knowledge, attitude contribute to putting them in vulnerable situations. If an effective management of such situation is not implemented, it may lead to the propagation of disease pathogens within and out of the affected site. These conditions are mainly related to status of water, sanitation and hygiene (WASH) in shelters and the living environment where disaster evacuees have settled; and the threat of occurrence of such epidemics is greater when evacuation sites are crowded. Improving WASH services is indispensable to prevent or control epidemics of communicable diseases in affected areas.

\section{Ways to enhance preparedness for communicable disease epidemic in Nepal}

An onsite inspection and fieldwork by the team in remote areas of those districts in the last trimester of the year 2015 made us realize that a number of disaster-affected areas have not been covered in terms of relief health care services. Diseased evacuees mostly do not benefit from any medical assistance and, in areas without a rural primary health center, they rarely visit health care providers who are based in health settings. Moreover, pregnant evacuees give birth in village shelters built by villagers themselves. Such health and security issues encountered by disaster-affected communities in remote areas were not monitored and an occurrence of CD cases could threaten the entire health of the local communities and neighboring districts.

In the disaster recovery phase, following this 2015 Nepal earthquake, the potential for the occurrence of CD epidemic is still present, given changes in ecology, economy, nutritional and health status of affected populations brought up by the disaster. This is a crucial period in which nurses might be helpful in accompanying the affected communities in their daily life, monitor their needs and health issues, and contribute to improving their living conditions and environment. The shelter status gets worse as time goes on if there are no solutions of recovery. Other interventions susceptible to enhance preparedness for CD epidemics should include:

- Improving the management of waste water, solid wastes disposal in the living environment;

- Conducting a systematic and comprehensive risk assessment in all disaster-affected areas and evacuation sites can identify time-sensitive factors that cause CD epidemic.

This would require implementation of participative community health education sessions for disaster-affected communities targeting the WASH sector in particular to induce healthy behaviors, such as avoiding coming to a contact with open defecation, digging holes to build acceptable toilets 
or install septic tanks and drainage systems whenever possible, putting public toilets in a reasonable distance from shelters, washing hands after using toilet and before meals, and drinking only safe or chlorinated water. It is equally urgent to apply geospatial technology for people's behavior modelling in disaster situations and to explore the effectiveness of the use of social network to enhance the understanding of the health security level of people in shelters. Development of trans-community preparation for comparative decision making for prompt disaster response is also an urgent task for public health security (Figure 1).

The relief teams from outside the country and the local government, should collaborate with local nurses who work, live in the community and are familiar with the conditions of the affected areas. The cultural gap between the survivor and the disaster responders requires implementing a flexible communication channel. Being sensitive to unique cultural behaviors and traits will increase the understanding, appreciation, and respect for cultural differences and similarities within, among, and between disaster relief groups. The Nepali local nurses from EpiNurse gathered first-hand information from evacuees in shelters and transmit data in near real-time communication to other health organizations that would make informed decisions for prevention of communicable diseases before an outbreak occurs, thanks to the action research which assesses living and environmental conditions and health risks, to improve the quality of reporting system in shelters, to enhance organizational development through teamwork around data collection, and to improve the quality of care by linking community and government surveillance records. This insight might contribute to development and continued enhancement of sustainability in the local communities. This solutionoriented activities challenge the conventional public health security monitoring system, which has required near real-time and population-based statistical alarms to issue alert to unusual activity, by offering a strong interactive network which crosses cultural and societal differences, geographies and generations (Figure 1).

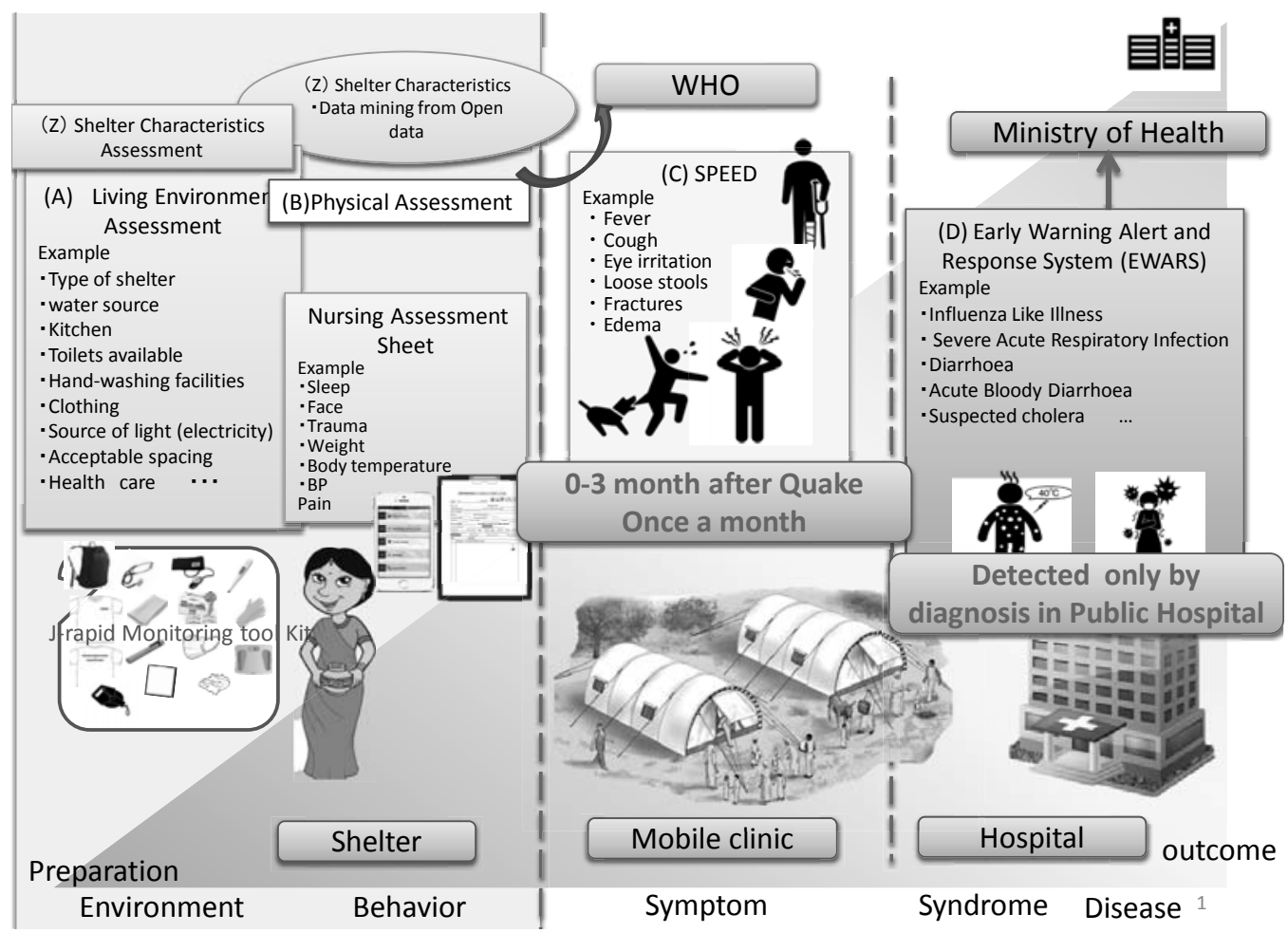

Figure 1. Pathogenesis \& Surveillance system 
In conclusion, periodical onsite risk assessment and monitoring of the effectiveness of actions and interventions implemented by delegates from the government and health clusters would contribute to improving preparedness for outbreaks of communicable diseases. Subsequently, on May 26, 2016 WHO Member States established a new Health Emergencies Program. This program adds operational capabilities for outbreaks and humanitarian emergencies and complements traditional roles. These new programs are designed to deliver rapid comprehensive support to countries and communities affected by disasters or conflicts, representing the need for risk reduction around the world. This idea would respond to the global agenda using a holistic approach for global collaboration that implements the accumulated knowledge. With formal education and drilling, competency can be developed in public health, enabling local nurses to make decision and create a system that would allow smooth cooperation among stakeholders. In local communities, nurses collect information about and mitigate communicable diseases risks. The data produced and their improved quality would confirm the claim by others that this information can assist with allocation of resources, the establishment of budgetary, long-term planning and productivity measurement and allotment of the work depending on literacy and skills of the community.

Furthermore, effective leadership is also necessary to manage improvement in reconstruction settings. Nurses are critically needed leaders in emergency management and disaster preparedness. They can also play a vital role in the restoration of public health under disaster condition and the identification of high risk and vulnerable population including unique needs of children in disaster.

\section{References}

Abou-Gareeb, A. H. (1961). Cholera in Nepal 1958-1960. Bull World Health Organ, 25(1): 130-134. Bhandari, D., Tandukar, S., Parajuli, H., Thapa, P., Chaudhary, P., Shrestha, D., ... , Sherchand, J. B. (2015). Cyclospora infection among school children in Kathmandu, Nepal: Prevalence and associated risk factors. Trop Med Health, 43(4): 211-216.

Department of Health Services (DOHS) of Nepal. (2016). Annual report 2015-2016. Retrieved from: http://dohs.gov.np/wp-content/uploads/2017/06/DoHS_Annual_Report_2072_73.pdf

Gautam, S., Jha, P., Khanal, B., Tamrakar, D., Yadav, D. K. (2012). Nepal: Cholera: Small Outbreak in Winter Season of Eastern Nepal. N Am J Med Sci, 4(12): 657-658.

Government of Nepal. Early warning and reporting system (EWARS), Weekly Bulletin, 7 September 2015. Retrieved from: https://www.humanitarianresponse.info/system/files/documents/files/ ewars_weekly_bulletin_34th_week.pdf

Government of Nepal, Ministry of Health and Population. (2015). Early Warnings and Reporting System Weekly Bulletin. September 7, 2015.

Katz, J. M. (2010). UN probes base a source of Haiti cholera outbreak. Retrieved from: http://www. washingtontimes.com/news/2010/oct/27/un-probes-base-as-source-of-haiti-cholera-outbreak/

Michel, R., Demoncheaux, J. P., Boutin, J. P., Baudon, D. (2007). Risk for epidemics after natural disasters. Emerg Infect Dis, 13(5): 785-786.

Noji, E. (1997). Public health consequences of disasters. New York: Oxford University Press.

Simkhada, P., van Teijlingen, E., Pant, P.R., Sathian, B., Tuladhar, G. (2015). Public health, prevention and health promotion in post-earthquake Nepal. Nepal J. Epidemiol, 5, 462-464.

Spiegel, P. B. (2005). Differences in world responses to natural disasters and complex emergencies. JAMA, 293: 1915-1918. 
Uchida, R., Pandey, B. D., Sherchand, J. B, Ahmed, K., Yokoo, M., Nakagomi, T., ..., Nakagomi, O. (2006). Molecular Epidemiology of Rotavirus Diarrhea among Children and Adults in Nepal: Detection of G12 Strains with P[6] or P[8] and a G11P[25] Strain. J Clin Microbiol, 44(10): 3499-3505.

Uprety, S., Hong, P.Y., Sadik, N., Dangol, B., Adhikari, R., Jutla, A., Shisler, J.L., Degnan, P., Nguyen, T.H. (2017). The effect of the 2015 earthquake on the bacterial community compositions in water in Nepal. Front Microbiol, 8, 2380.

Uprety, S., Iwelunmor, J., Sadik, N., Dangol, B., Nguyen, T. H. (2017). A qualitative case study of water, sanitation, hygiene resources after Gorkha earthquake in Nepal. Earthquake Spectra, 33(S1): S133-S146.

Watson, J. T., Gayer, M., Connolly, M. A. (2007). Epidemics after natural disasters. CDC (Center for Disease Prevention and Control) report, January 2007. Retrieved from: http://wwwnc.cdc. gov/eid/article/13/1/06-0779_article

World Health Organization (WHO). (2015). Nepal: Country profile. Retrieved from: http://www. who.int/gho/countries/npl.pdf?ua=1

\section{Author Profile}

Dr. Sakiko Kanbara is Associate Professor at the University of Kochi, Japan, since 2012.
She graduated from the Faculty of Medicine of the University of Kobe in 2000, got her Ph.D
degree in Public Health at Okayama University Medical School, Japan (2012). She worked
as a researcher at the University of Hyogo, Japan, and studied disaster Nursing and Global
Nursing. She conducted several collaborative research projects in the Philippines, Indonesia,
and Nepal. In 2015, she created the 'EpiNurse research team' for global nursing and disaster
risk reduction. She also served as secretary of World Society of Disaster Nursing.

